\title{
Efeito da covariância genética aditivo-materna sobre estimativas de parâmetros genéticos e em avaliações genéticas de características de crescimento de animais Tabapuã
}

\author{
[Effect of additive genetic-maternal covariance on genetic parameter estimates and on genetic evaluation \\ of growth traits of Tabapuã Zebu breed]

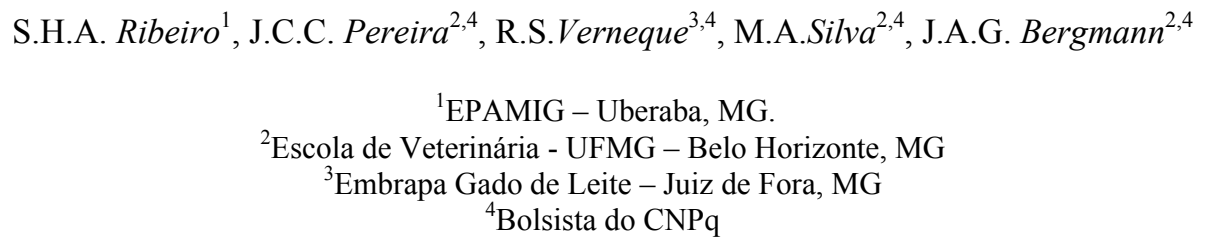 \\ RESUMO
}

Avaliou-se o efeito da covariância genética aditiva direto-materna sobre estimativas de parâmetros genéticos e sobre a predição e a ordenação de valores genéticos de animais da raça Tabapuã. Os parâmetros genéticos foram estimados com base em 19646, 14276, 10663 e 6172 registros de pesos ao nascimento e ajustados para 205, 365 e 550 dias de idade em análises unicaracterística, utilizando o programa computacional MTDREML, pelo método da máxima verossimilhança restrita livre de derivadas por modelos animal com ou sem a inclusão da covariância. As estimativas da covariância genética aditiva direto-materna foram, respectivamente, $-0,08 ;-0,22 ;-0,10$ e 0,34 , para os pesos ao nascer e ajustados para 205, 365 e 550 dias de idade. As herdabilidades diretas e maternas obtidas sob modelo com a inclusão da covariância genética direto-materna foram, respectivamente, 0,31 e 0,$10 ; 0,20$ e 0,$17 ; 0,20$ e 0,06 e 0,17 e 0,01 , para os mesmos pesos, enquanto sem a inclusão foram, respectivamente, 0,31 e 0,$09 ; 0,18$ e 0,14 ; 0,20 e 0,05 e 0,18 e 0,02 . Os valores de correlação de posto entre os valores genéticos preditos pelos modelos com e sem a inclusão foram 0,999; 0,992; 0,999 e 0,998. As correlações de posto entre os valores genéticos maternos foram 0,$999 ; 0,985 ; 0,992$ e 0,771 . A inclusão da covariância genética aditiva direto-materna não influenciou as estimativas dos parâmetros genéticos e teve efeito inexpressivo na ordenação dos valores genéticos dos pesos de animais da raça Tabapuã.

Palavras-chave: gado de corte, Tabapuã, covariância aditivo-materna, avaliação genética

\begin{abstract}
This study was carried out to evaluate the effect of additive genetic-maternal covariance on estimates of genetic parameters and on the prediction and ranking of breeding values of Tabapuã animals. Records of 19,646, 14,276, 10,663, and 6,176 birth weights and weights at 205, 365, and 550 days of age were used in univariate animal model analysis using MTDFREML software, including or not the additive geneticmaternal covariance in the models. The additive genetic-maternal covariance estimates were, respectively, $-0.08,-0.22,-0.10$, and 0.34 for birth weight and weights at 205, 365, and 550 days of age. The direct and maternal heritability estimates including the additive direct-maternal covariance were, respectively, 0.31, and 0.10; 0.31 and 0.20, and 0.17; and 0.20 and 0.06; and 0.17 and 0.01 for birth weight and weights at 205, 365, and 550 days of age, while without the additive direct-maternal covariance those values were, respectively, 0.31 and $0.09 ; 0.18$ and $0.14 ; 0.20$ and $0.05 ; .18$ and 0.02 . Rank correlation between predicted breeding values from two models for birth weight and weights at 205 and 550 days of age were, respectively, 0.999, 0.992, 0.999, and 0.998. For maternal genetic values, these estimated rank correlations were, respectively, 0.995, 0.985, 0.992, and 0.771. The inclusion of additive genetic-maternal covariance in the analysis did not affect genetic parameter estimates and had a very small effect on breeding values ranking of Tabapuã animals.
\end{abstract}

Keywords: beef cattle, Tabapuã, additive-maternal covariance, genetic evaluation

Recebido em 30 de outubro de 2008

Aceito em 4 de fevereiro de 2009

E-mail: sandro.ribeiro@epamiguberaba.com.br 


\section{INTRODUÇÃO}

Os pesos ao nascer e à desmama são reconhecidamente características indicadoras, pelo menos parcialmente, dos efeitos maternos. A importância do efeito materno durante a fase de crescimento pré-desmama tem como sustentação fisiológica a habilidade de a fêmea prover as necessidades mínimas capazes de permitir a sobrevivência e o desenvolvimento do seu produto. A literatura que trata do tema relata grande variação de amplitude das estimativas de covariância genética aditiva direto-materna nos pesos tomados às várias idades (Tab. 1). A existência de correlação genética negativa entre os efeitos genéticos diretos e maternos caracteriza o antagonismo entre os efeitos dos genes para potencial de crescimento e dos genes para a habilidade materna, notadamente para as características ponderais até os 365 dias de idade.

Tabela 1. Estimativas de parâmetros genéticos dos pesos ao nascer (PN), à desmama (P205), aos 365 (P365) e 550 dias de idade (P550) por autor, ano e raça

\begin{tabular}{|c|c|c|c|c|c|c|c|}
\hline \multirow{2}{*}{ Peso } & \multirow{2}{*}{ Raça } & \multirow{2}{*}{$\mathrm{N}$} & \multicolumn{4}{|c|}{ Parâmetro genético } & \multirow[b]{2}{*}{ Autores } \\
\hline & & & $\mathrm{h}_{\mathrm{o}}^{2}$ & $h_{m}^{2}$ & $\mathrm{r}_{\mathrm{am}}$ & $c^{2}$ & \\
\hline \multirow[t]{7}{*}{$\mathrm{PN}$} & $\mathrm{H}$ & 5488 & 0,41 & 0,08 & 0,04 & 0,05 & Meyer (1992) \\
\hline & A & 4036 & 0,36 & 0,07 & 0,28 & 0,03 & Meyer (1992) \\
\hline & $\mathrm{H}$ & 2532 & 0,24 & 0,11 & 0,37 & 0,03 & Waldron et al (1993) \\
\hline & $\mathrm{A}$ & 3323 & 0,33 & 0,04 & 0,28 & 0,06 & Waldron et al (1993) \\
\hline & $\mathrm{N}$ & 27549 & 0,22 & 0,12 & $-0,72$ & 0,07 & Eler et al. (1995) \\
\hline & A & 5747 & 0,47 & 0,17 & $-0,52$ & 0,07 & Robinson (1996) \\
\hline & $\mathrm{T}$ & 20586 & 0,31 & 0,11 & $-0,28$ & $*$ & Fridrich et al. (2003) \\
\hline \multirow[t]{8}{*}{ P205 } & $\mathrm{H}$ & 7003 & 0,14 & 0,13 & $-0,58$ & 0,23 & Meyer (1992) \\
\hline & A & 3465 & 0,20 & 0,14 & 0,22 & 0,03 & Meyer (1992) \\
\hline & M & 2842 & 0,58 & 0,36 & $-0,78$ & 0,11 & Meyer (1992) \\
\hline & $\mathrm{H}$ & 2395 & 0,15 & 0,14 & $-0,35$ & 0,21 & Waldron et al (1993) \\
\hline & A & 3149 & 0,14 & 0,11 & 0,06 & 0,15 & Waldron et al (1993) \\
\hline & $\mathrm{N}$ & 27549 & 0,13 & 0,13 & $-0,32$ & 0,14 & Eler et al. (1995) \\
\hline & A & 13376 & 0,26 & 0,15 & $-0,45$ & 0,14 & Robinson (1996) \\
\hline & $\mathrm{T}$ & 19482 & 0,15 & 0,20 & $-0,18$ & $*$ & Fridrich et al. (2003) \\
\hline \multirow[t]{8}{*}{ P365 } & $\mathrm{H}$ & 1449 & 0,16 & 0,11 & $-0,48$ & 0,05 & Meyer (1992) \\
\hline & $\mathrm{A}$ & 2374 & 0,33 & 0,04 & 0,48 & 0,02 & Meyer (1992) \\
\hline & M & 2480 & 0,25 & 0,14 & $-0,39$ & 0,02 & Meyer (1992) \\
\hline & $\mathrm{H}$ & 2216 & 0,28 & 0,01 & 0,97 & 0,06 & Waldron et al (1993) \\
\hline & A & 2927 & 0,30 & 0,08 & 0,04 & 0,00 & Waldron et al (1993) \\
\hline & $\mathrm{N}$ & 27549 & 0,16 & 0,10 & 0,09 & 0,02 & Eler et al. (1995) \\
\hline & A & 6292 & 0,28 & 0,11 & $-0,31$ & 0,06 & Robinson (1996) \\
\hline & $\mathrm{T}$ & 13796 & 0,19 & 0,06 & 0,01 & $*$ & Fridrich (2003) \\
\hline \multirow[t]{5}{*}{ P550 } & $\mathrm{H}$ & 2783 & 0,22 & 0,03 & $-0,20$ & 0,09 & Meyer (1992) \\
\hline & M & 2121 & 0,20 & 0,005 & 1,00 & 0,05 & Meyer (1992) \\
\hline & $\mathrm{H}$ & 1508 & 0,38 & 0,01 & 0,95 & 0,01 & Waldron et al (1993) \\
\hline & A & 1590 & 0,20 & 0,08 & 0,39 & 0,00 & Waldron et al (1993) \\
\hline & A & 5268 & 0,29 & 0,05 & $-0,37$ & 0,05 & Robinson (1996) \\
\hline
\end{tabular}

$\mathrm{h}^{2}$ : herdabilidade aditiva direta; $\mathrm{h}^{2}{ }_{\mathrm{m}}$ : herdabilidade aditiva materna; $\mathrm{r}_{\mathrm{am}}$ : correlação genética entre os efeitos maternos e diretos; $\mathrm{c}^{2}$ : efeito de meio permanente; N: Nelore; M: mestiço Zebu X Bos taurus taurus; H: Hereford; A: Angus; T: Tapapuã. 
Waldron et al. (1993) sugeriram em 1993 a competição metabólica por nutrientes como origem para a covariância genética aditivomaterna negativa. Esses autores justificaram que, em ambientes limitantes, em razão da escassez, os nutrientes são dirigidos para uma ou outra produção, no caso em questão, para a produção de carne ou leite, fator responsável por $60 \%$ da expressão materna. Robinson (1996) sugeriu a não contabilização da variação adicional promovida pela adição de touros de alto valor genético, com pedigrees ignorados e oriundos principalmente da importação, em rebanhos comerciais, assim como, a influência do fator interação touro e ano. Maniatis e Pollott (2003) pesquisaram o impacto que a estrutura dos dados exercia sobre a estimação dos componentes de (co)variância das características de crescimento em carneiros. Observaram que a magnitude da correlação genética aditiva direto-negativa declinava à medida que se aumentava nos dados o número de mães com registros próprios de produção e com maior número de progênies. Concluíram como causa primária da negativa e biologicamente impossível correlação genética aditivo-materna a utilização de banco de dados limitados.

Assim, este trabalho teve o objetivo de avaliar possíveis influências da covariância genética direto-materno sobre a estimação dos parâmetros genéticos e predição dos valores genéticos quando adicionada nas análises.

\section{MATERIAL E MÉTODOS}

Foram avaliados $19.646,14.276,10.663$ e 6.172 registros, respectivamente, de pesos ao nascimento e pesos ajustados para 205, 365 e 550 dias de idade. Estes dados foram cedidos pela Associação Brasileira de Criadores de Zebu (ABCZ), como resultado do programa de controle ponderal realizado pelo Serviço de Controle de Desenvolvimento Ponderal (CDP) dessa instituição na fazenda Água Milagrosa, município de Tabapuã, São Paulo, no período de 1978 a 2002.

$\mathrm{Na}$ formação dos arquivos e consistência dos dados, foram excluídos da análise, em razão do baixo número, os registros dos anos anteriores a 1978 e posteriores a 2002, os registros dos pesos ao nascimento e aos 550 dias de idade, respectivamente, menores que 21 e $100 \mathrm{~kg}$ e maiores que 42 e $500 \mathrm{~kg}$, e os registros com ordem de parto maior que 13 . Os registros com ordem de parto entre 10 e 13 foram agrupados em um único grupo com o intuito de eliminar dados que geram pouca informação e muito viés, animais sem registro de pai e mãe, reprodutores acasalados com menos de três fêmeas e fêmeas com menos de três crias também foram excluídos da análise. Para a formação de arquivos e consistência, foi utilizado pacote estatístico SAS/1998.

Os efeitos fixos ano e época de nascimento, sexo e ordem de parto foram considerados na análise para todos os pesos, com exceção do peso aos 550 dias de idade em que a ordem de parto não apresentou influência significativa $(\mathrm{P}>0,05)$.

O programa estatístico MTDFREML, desenvolvido por Boldman et al. (1995), foi utilizado para análise descritiva e estimação dos componentes de (co)variância pelo método da máxima verossimilhança restrita com o procedimento livre de derivadas sob modelo animal, utilizando-os, posteriormente, no cálculo dos correspondentes parâmetros genéticos e predição dos valores genéticos. $\mathrm{O}$ método simplex, proposto por Nelder e Mead (Boldman et al., 1995), foi utilizado pelo programa para localizar o mínimo de $-2 \log \mathrm{L}$, em que $\mathrm{L}$ representa a função de verossimilhança depois de atingido o critério de convergência $\left(10^{-9}\right)$.

O modelo estatístico foi:

$\mathrm{y}=\mathrm{X} \beta+\mathrm{Z} \mu+\mathrm{Wm}+\mathrm{Spe}+\mathrm{e}, \mathrm{em}$ que $:$

$\mathrm{y}=$ vetor de observações das variáveis dependentes (pesos aos nascer, à desmama, aos 365 e 550 dias de idade);

$\beta=$ vetor de efeitos fixos (ano época, sexo e ordem de parto);

$\mu=$ vetor dos efeitos genéticos aditivos de cada um dos indivíduos (efeitos aleatórios);

$\mathrm{m}=$ vetor dos efeitos genéticos maternos;

$\mathrm{pe}=$ vetor dos efeitos de meio permanentes sobre os produtos de cada vaca; $\mathrm{e}=$ vetor de efeitos aleatórios residuais.

$\mathrm{X}, \mathrm{Z}, \mathrm{W}$ e S são matrizes de incidência relacionadas, respectivamente, aos efeitos fixos do animal, genético-materno e de ambiente permanente. Foram realizadas duas análises unicaracterística para os pesos ao nascer, aos 
205, 365 e 550 dias de idade, com a inclusão ou não da covariância genética aditiva diretomaterna.

\section{RESULTADOS E DISCUSSÃO}

As médias, desvios-padrão, coeficientes de variação e amplitudes e valores mínimo e máximo dos pesos estudados são apresentados na Tab. 2. Os valores médios e desvios-padrão estimados foram próximos aos encontrados por Fridrich et al. (2005), que estudou essas mesmas características em rebanhos da raça Tabapuã em várias regiões do Brasil.

Tabela 2. Números de observações, médias e desvios-padrão (DP), coeficientes de variação (CV\%) e amplitudes observadas para os pesos de bovinos Tabapuã

\begin{tabular}{lcccccc}
\hline \multicolumn{1}{c}{ Característica } & $\mathrm{N}$ & Média & DP & CV & Min & Max \\
\hline Peso ao nascer & 19646 & 30,83 & 3,28 & 10.65 & 21,00 & 42,00 \\
Peso à desmama & 14276 & 172,53 & 21,55 & 12,49 & 61,00 & 368,00 \\
Peso aos 365 dias de idade & 10663 & 221,94 & 29,82 & 13,44 & 133,00 & 353,00 \\
Peso aos 550 dias de idade & 6172 & 281,25 & 37,86 & 13,46 & 159,00 & 452,00 \\
\hline
\end{tabular}

DP: desvio-padrão; CV: coeficiente de variação; Min: valor mínimo; Max: valor máximo.

Os componentes de (co)variância e os parâmetros genéticos dos pesos ao nascimento, aos 205,365 e 550 dias de idade estimados em análises unicaracterística com ou sem a inclusão da covariância entre os efeitos genéticos direto e materno são apresentados na Tab. 3.

Os componentes de (co)variância e os parâmetros genéticos, obtidos nas análises com a inclusão da covariância genética direto-materna, foram semelhantes aos de Fridrich et al. (2005). Contudo, em seu estudo a autora não adicionou a variável ambiente permanente nas análises, o que, de certa forma, é confirmado neste estudo, já que sua inclusão não foi importante nem na expressão dessas características nem na determinação dos outros componentes de variação.

Como já visto em outros trabalhos (Tab. 1), a maior influência do efeito materno deu-se no peso à desmama, embora sua influência já fosse observada desde o peso ao nascimento, o que segundo Kock Clark (1955), se deve à qualidade do ambiente uterino. Houve decréscimo da influência materna nos pesos posteriores à desmama, que chegou a explicar apenas $1 \%$ da variação total no peso aos 550 dias de idade. Em todos os pesos, observou-se correlação genética direto-materna negativa, à exceção do peso aos 550 dias de idade. $\mathrm{O}$ peso à desmama apresentou a correlação negativa de maior magnitude.
Nas análises que não incluíram a covariância genética direto-materna, houve acréscimo no componente de variância do erro e redução nos demais componentes de variância em todos os pesos à exceção do peso aos 550 dias de idade em que aconteceu o inverso. Esta é coerente em razão da diferença entre os sinais da correlação genética direto-materna desse com as dos demais pesos. Alterações nos componentes de variância não refletiram nos parâmetros genéticos em função de a variação fenotípica também ter sido reduzida. Observou-se redução moderada das herdabilidades direta e materna do peso à desmama, em menor intensidade nas herdabilidades materna dos pesos ao nascimento e aos 365 dias de idade, e ligeiro aumento das herdabilidades direta e materna do peso aos 550 dias de idade, que levaram pequenas modificações às relativas herdabilidades totais, índice proposto por Willham (1972), que representa a regressão do valor genético total do animal (efeitos direto e materno) em relação ao seu fenótipo. As herdabilidades diretas para o peso ao nascer e aos 365 dias de idade não se alteraram quando não se incluiu a covariância genética aditiva direto-materna nas análises.

$\mathrm{Na}$ Tab. 4 são apresentados os coeficientes de correlação de posto de Spearman entre os valores genéticos preditos com (modelo 1) e sem (modelo 2) a inclusão da covariância genética direto-materna nas análises 
Efeito da covariância genética...

Tabela 3. Estimativas dos componentes de (co)variância genética aditiva, materna e parâmetros genéticos dos pesos ao nascer (PN), aos 205 (P205), 365 (P365) e 550 (P550) dias de idade obtidas por análise unicaracterística

\begin{tabular}{lcccccc}
\hline \multirow{2}{*}{ Característica } & \multicolumn{7}{c}{ Componente de variância } \\
\cline { 2 - 7 } & $\sigma_{\mathrm{p}}^{2}$ & $\sigma_{\mathrm{a}}^{2}$ & $\sigma_{\mathrm{m}}^{2}$ & $\sigma_{\mathrm{am}}$ & $\sigma_{\mathrm{c}}^{2}$ & $\sigma_{\mathrm{e}}^{2}$ \\
\hline Peso ao nascer & 9,85 & 3,09 & 0,96 & $-0,15$ & 0,00 & 5,94 \\
& 9,84 & 3,01 & 0,85 & 0,00 & 0,00 & 5,98 \\
\hline Peso à desmama & 310,49 & 62,86 & 52,58 & $-12,51$ & 0,00 & 207,57 \\
& 310,28 & 56,43 & 42,77 & 0,00 & 0,00 & 210,91 \\
\hline Peso aos 365 dias de idade & 443,07 & 90,47 & 27,16 & $-4,76$ & 0,00 & 330,19 \\
& 442,89 & 87,41 & 23,74 & 0,00 & 0,00 & 331,73 \\
\hline Peso aos 550 dias de idade & 854,52 & 146,05 & 9,54 & 12,54 & 0,00 & 686,39 \\
& 854,26 & 150,22 & 19,50 & 0,00 & 0,00 & 684,54 \\
\hline \multicolumn{1}{c}{ Característica } & & & Parâmetro genéticos & & \\
\hline Peso ao nascer & $\mathrm{h}_{\mathrm{T}}^{2}$ & $\mathrm{~h}_{\mathrm{a}}^{2}$ & $\mathrm{~h}_{\mathrm{m}}^{2}$ & $\mathrm{r}_{\mathrm{am}}$ & $\mathrm{C}^{2}$ & $\mathrm{E}^{2}$ \\
\hline Peso à desmama & 0,34 & 0,31 & 0,10 & $-0,08$ & 0,00 & 0,60 \\
& 0,35 & 0,31 & 0,09 & 0,00 & 0,00 & 0,61 \\
\hline Peso aos 365 dias de idade & 0,23 & 0,20 & 0,17 & $-0,22$ & 0,00 & 0,67 \\
& 0,25 & 0,18 & 0,14 & 0,00 & 0,00 & 0,68 \\
\hline Peso aos 550 dias de idade & 0,22 & 0,20 & 0,06 & $-0,10$ & 0,00 & 0,75 \\
& 0,22 & 0,20 & 0,05 & 0,00 & 0,00 & 0,75 \\
\hline
\end{tabular}

$\sigma_{\mathrm{p}}^{2}$ : variância fenotípica; $\sigma_{\mathrm{a}}^{2}$ : variância aditiva direta; $\sigma_{\mathrm{m}}^{2}$ : variância aditiva materna; $\sigma_{\mathrm{m}}$ : covariância aditiva diretomaterna; $\sigma_{\mathrm{c}}^{2}$ : variância de meio permanente: $\sigma_{\mathrm{e}}^{2}$ : variância residual; $\mathrm{h}_{\mathrm{T}}^{2}=\sigma_{\mathrm{a}}^{2}+1,5 \sigma_{\mathrm{am}}+0,5 \sigma_{\mathrm{m}}^{2} / \sigma_{\mathrm{p}}^{2}$ (Willham, 1972); $\mathrm{h}_{\mathrm{a}}^{2}:$ herdabilidade direta; $\mathrm{h}_{\mathrm{m}}^{2}:$ herdabilidade materna; $r_{a m}$ : correlação genética aditiva direto-materna: $\mathrm{C}^{2}$ : fração de meio permanente; $E^{2}$ fração do resíduo.

Tabela 4. Estimativas de coeficientes de correlação de Spearman para os pesos ao nascer, à desmama, aos 365 e 550 dias de idade

\begin{tabular}{|c|c|c|c|c|c|c|c|c|c|c|}
\hline \multirow{3}{*}{\multicolumn{3}{|c|}{$\begin{array}{l}\text { Coeficiente de correlação de } \\
\text { Spearman }\end{array}$}} & \multicolumn{8}{|c|}{ Modelo 1} \\
\hline & & & \multicolumn{2}{|c|}{ PN } & \multicolumn{2}{|c|}{ P205 } & \multicolumn{2}{|c|}{ P365 } & \multicolumn{2}{|c|}{ P550 } \\
\hline & & & VGD & VGM & VGD & VGM & VGD & VGM & VGD & VGM \\
\hline \multirow{8}{*}{$\frac{N}{\frac{0}{0}}$} & \multirow{2}{*}{ PN } & VGD & 0,999 & $*$ & $*$ & $*$ & $*$ & $*$ & $*$ & $*$ \\
\hline & & VGM & $*$ & 0,995 & $*$ & $*$ & $*$ & $*$ & $*$ & $*$ \\
\hline & \multirow{2}{*}{ P205 } & VGD & $*$ & $*$ & 0,992 & $*$ & $*$ & $*$ & $*$ & $*$ \\
\hline & & VGM & $*$ & $*$ & $*$ & 0,985 & $*$ & $*$ & $*$ & $*$ \\
\hline & \multirow{2}{*}{ P365 } & VGD & $*$ & $*$ & $*$ & $*$ & 0,999 & $*$ & $*$ & $*$ \\
\hline & & VGM & $*$ & $*$ & $*$ & $*$ & $*$ & 0,992 & $*$ & $*$ \\
\hline & \multirow{2}{*}{ P550 } & VGD & $*$ & $*$ & $*$ & $*$ & $*$ & $*$ & 0,998 & $*$ \\
\hline & & VGM & * & * & * & * & * & $*$ & * & 0,771 \\
\hline
\end{tabular}

PN: peso ao nascer; P205: peso à desmama; P365 e P550: peso aos 365 e 550 dias de idade respectivamente; VGD: valor genético aditivo direto; VGM: valor genético aditivo-materno. 
Os coeficientes foram muito próximos à unidade em todas as características, o que pode ser visualizado pela alta porcentagem de animais coincidentes dentro dos 20 mais altos valores genéticos para cada característica, quando as avaliações com e sem a inclusão da covariância direto-materna são comparadas (Tab. 5), o que sugere que a inclusão da covariância genética aditivo direto-materna nos modelos é irrelevante para predição dos valores genéticos e correta classificação e seleção dos melhores animais.

Tabela 5. Porcentagem de animais que coincidiram estar dentro dos 20 melhores valores genéticos para cada característica

\begin{tabular}{cccc}
\hline \multicolumn{5}{c}{ VGD } & \\
\hline PN & P205 & P365 & P550 \\
\hline 0,95 & 0,95 & 1,00 & 0,95 \\
\hline PN & VGM & & \\
\hline 0,95 & P205 & P365 & P550 \\
\hline
\end{tabular}

VGD: valor genético direto; VGM: valor genético materno.

\section{CONCLUSÃO}

Não há necessidade da inclusão da covariância genética aditiva direto-materna em análises de avaliações genéticas das características de crescimento de animais da raça Tabapuã.

\section{AGRADECIMENTOS}

À Fazenda Água Milagrosa, em especial ao Sr. Carlos Arthur Ortenblad, por consentir no uso do banco de dados dessa propriedade. À Associação Brasileira de Criadores de Zebu (ABCZ), em especial ao Sr. Luiz Antonio Josahkian, superintendente técnico, e Sr. Rodemir Urbano dos Santos, gerente de desenvolvimento, pela presteza na informatização e disponibilização dos dados. À Coordenadoria de Aperfeiçoamento de Pessoal de Ensino Superior (CAPES), pelo suporte financeiro prestado no decorrer do estudo.

\section{REFERÊNCIAS BIBLIOGRÁFICAS}

BOLDMAN, K.G.; KRIESE, L.A.; VAN VLECK, L.D. et al. A manual for use of MTDFREML: a set of programs to obtain estimates of variances and covariances (DRAFT) Lincoln: Department of Agriculture/Agricultural Research Service, 1995. 125p.

ELER, J.P.; VAN VLECK, L.D.; FERRAZ, J.B.E. et al. Estimation of variances due to direct and maternal effects for growth traits of Nelore cattle. J. Anim. Sci., v.73, p.3253-3258, 1995.
FRIDRICH, A.B.; SILVA, M.A.; FRIDRICH, D. et al. Interação genótipo $\mathrm{x}$ ambiente e estimativas de parâmetros genéticos de características ponderais de bovinos Tabapuã. Arq. Bras. Med. Vet. Zootec., v.57, p.663-672, 2005.

KOCH, R.M.; CLARK, R.T. Genetic and ernvironmental relationships among economic characters in beef cattle. III. Evaluating maternal enviroment. J. Anim. Sci., v.14, p.979-985, 1955.

MANIATIS, N.; POLLOTT, G.E. The impact of data structure on genetic (co)variance components of early growth in sheep, estimated using an animal model with maternal effects. $J$. Anim. Sci., v.81, p.101-108, 2003.

MEYER, K. Variance components due to direct and maternal effects for growth traits of Australian beef cattle. Livest. Prod. Sci., v.31, p.179-204, 1992.

ROBINSON, D.L. Estimation and interpretation of direct and maternal genetic parameters for weights of Australian Angus cattle. Livest. Prod. Sci., v.45, p.1-11, 1996.

WALDRON, D.F.; MORRIS, C. A.; BAKER, R. L. et al. Maternal effects for growth traits in beef cattle. Livest. Prod. Sci., v.34, p.57-70, 1993.

WILHAM, R.L. The role of maternal effects in animal breeding: III. Biometrical aspects of maternal effects in animals. J. Anim. Sci., v.35, p.1288-1293, 1972. 